\title{
Eichler cohomology and zeros of polynomials associated to derivatives of $L$-functions
}

\author{
Nikolaos Diamantis (University of Nottingham) \\ Larry Rolen (Vanderbilt University)
}

April 14, 2020

\begin{abstract}
In recent years, a number of papers have been devoted to the study of zeros of period polynomials of modular forms. Here, we study cohomological analogues of the EichlerShimura period polynomials corresponding to higher $L$-derivatives. We state a general conjecture about the locations of the zeros of the full and odd parts of the polynomials, in analogy with the existing literature on period polynomials, and we also give numerical evidence that similar results hold for our higher derivative "period polynomials" in the case of cusp forms. The unimodularity of the roots seems to be a very subtle property which is special to our "period polynomials". This is suggested by numerical experiments on families of perturbed "period polynomials" (Sect. 5.3) suggested by Zagier. We prove a special case of our conjecture in the case of Eisenstein series.

Although not much is currently known about derivatives higher than first order ones for general modular forms, celebrated recent work of Yun and Zhang established the analogues of the Gross-Zagier formula for higher $L$-derivatives in the function field case. A critical role in their work was played by a notion of "super-positivity", which, as recently shown by Goldfeld and Huang, holds in infinitely many cases for classical modular forms. As will be discussed, this is similar to properties which were required by Jin, Ma, Ono, and Soundararajan in their proof of the Riemann Hypothesis for Period Polynomials, thus suggesting a connection between the analytic nature of our conjectures here and the framework of Yun and Zhang.
\end{abstract}

\section{Introduction}

Derivatives of $L$-functions, and especially higher order derivatives, remain mysterious objects. This is despite intense work on key conjectures about them, such as those of Beilinson, BirchSwinnerton-Dyer, etc. Beilinson's conjecture, as formulated in [23], relates values of derivatives of $L$-functions to fundamental objects called periods. In [23], a complex number is called a 
period if its real and imaginary parts have the form

$$
\int_{V} \frac{P(\mathbf{x})}{Q(\mathbf{x})} d \mathbf{x}
$$

where $V$ is a domain in $\mathbb{R}^{n}$ defined by polynomial inequalities with coefficients in $\mathbb{Q}$ and $P, Q \in \mathbb{Q}\left[X_{1}, \ldots, X_{n}\right]$. This definition accounts for numbers that are clearly very important for number theory and other areas of mathematics, but are not necessarily algebraic, e.g.

$$
\pi=\iint_{x^{2}+y^{2} \leq 1} d x d y \quad \text { and } \log (n)=\int_{1}^{n} \frac{1}{x} d x(\text { for } n \in \mathbb{N}) .
$$

Denote the set of periods by $\mathcal{P}$. A special case of Beilinson's conjecture in a version given in [23] can be stated as follows.

Conjecture (Deligne-Beilinson-Scholl). Let $f$ be a weight $k$ Hecke eigencuspform for $S L_{2}(\mathbb{Z})$, $L_{f}(s)$ its $L$-function, and $m$ an integer. Then, if $r$ is the order of vanishing of $L_{f}(s)$ at $s=m$, we have

$$
L^{(r)}(m) \in \mathcal{P}[1 / \pi]
$$

The cases of $r=0$ and $m=1, \ldots, k-1$ (that is, the case of critical values of $L_{f}(s)$ ) have been treated by Manin, Deligne and others (e.g. [25, 7]). The case when $r=0$ and $m>k-1$ has been proven by Beilinson and Deninger-Scholl (see [23] and the references therein) but for $r>0$ the picture is much less clear. Fundamental results by Gross-Zagier [17] and others in the context of the Birch-Swinnerton-Dyer conjecture give insight for $r=1$ and $k=2$. In the function field case, celebrated work of Yun and Zhang [31] gives the analogues of the Gross-Zagier formula for higher $L$-derivatives. However, in the number field case, very little is known for $r \geq 2$. Inspired by a notion of "super-positivity" introduced in YunZhang, Goldfeld and Huang [16] established that infinitely many modular forms have the same property of positivity of all of their central $L$-derivatives. As will be described in Section 5.2, this is similar to properties which were required by Jin, Ma, Ono, and Soundararajan in their proof of the Riemann Hypothesis for Period Polynomials [20]. Thus, these properties of Yun and Zhang seem related to the general conjectures we present here.

One of the important tools for studying critical values is the period polynomial

$$
Q_{f}(z)=Q_{f}(0 ; z):=\int_{0}^{\infty} f(\tau)(\tau-z)^{k-2} d \tau=\sum_{n=0}^{k-2}\left(\begin{array}{c}
k-2 \\
n
\end{array}\right) i^{1-n} \Lambda_{f}(n+1) z^{k-2-n}
$$

where $\Lambda_{f}(s):=(2 \pi)^{-s} \Gamma(s) L_{f}(s)$ denotes the completed $L$-function (e.g. [25, 22, 32]). Background for the period polynomial will be discussed in the next section.

In this paper, we offer the following conjecture about an analogue of this polynomial for all derivatives of $L_{f}(s)$ and then prove it in the case of Eisenstein series. This analogue is given for each $m \in \mathbb{N}^{*}$, by

$$
Q_{f}(m ; z):=\int_{0}^{\infty} f(\tau)(\tau-z)^{k-2} \log (\tau / i)^{m} d \tau=\sum_{n=0}^{k-2}\left(\begin{array}{c}
k-2 \\
n
\end{array}\right) i^{1-n} \Lambda_{f}^{(m)}(n+1) z^{k-2-n} .
$$


It can be written as a sum of its even part $Q_{f}^{+}(m ; z)$ and of its odd part $Q_{f}^{-}(m ; z)$. With this notation we can now state the following.

Conjecture 1.1. ("Riemann hypothesis for period polynomials attached to derivatives of Lfunctions")

i). For any Hecke eigenform of weight $k$ on $\mathrm{SL}_{2}(\mathbb{Z})$, and for each $m \in \mathbb{N}^{*}$, the polynomial $Q_{f}(m ; z)$ has all its zeros on the unit circle.

ii). The odd part $Q_{f}^{-}(m ; z)$ of $Q_{f}(m ; z)$ has a unique root $a_{f, m}>1$. Other than the 5 zeros at $0, \pm a_{f, m}, \pm 1 / a_{f, m}$, all roots of the odd part of the period polynomial lie on the unit circle.

Remark. It is natural to ask whether an analogous conjecture could be made for the even part of $Q_{f}(m ; z)$. The numerical tests we carried out indicate that there is no obvious pattern. For instance, for some $m$, the polynomial $Q_{\Delta}(m ; z)$ (for the weight $12 \Delta$-function) has 4 roots off the unit circle and for other $m$ it has 8 roots off the unit circle. We intend to investigate this phenomenon in future work.

By the work of J.B. Conrey, D.W. Farmer and Ö. Imamoğlu [6], part ii). of Conjecture 1.1 is true for $m=0$ with $a_{f, m}=2$. For $m \geq 1$, we have verified both parts in a number of initial cases. Specifically,

Proposition 1.2. Conjecture 1.1 is true for all derivatives $m \leq 3$ and for all newforms with weights $k \leq 50$.

We list the values for $a_{f, m}>1$ for Hecke eigenforms $f$ of weight $k=12,18,24$ and number of derivatives $m=1,2,3$.

Table 1: Numerical values for the unique root $a_{f, m}>1$ of odd parts of period polynomials

\begin{tabular}{|c|c|c|c|}
\hline$k \backslash m$ & 1 & 2 & 3 \\
\hline 12 & 1.70249485316 & 1.49532959768 & 1.41366616080 \\
\hline 18 & 1.87689145347 & 1.77925293389 & 1.69835493406 \\
\hline 24 & 1.93021754570 & 1.86841038048 & 1.81295686978 \\
\hline 24 & 1.93028942678 & 1.86847424264 & 1.81300019566 \\
\hline
\end{tabular}

Remark. The main obstacle to extending to $m \geq 1$ the proof of the corresponding results for $m=0$ given in [6, 27, 20] etc. is the lack of sufficient information for values of derivatives of $\Lambda_{f}(s)$. For instance, to apply Sturm's theorem to determine the number of real roots of $Q_{f}^{-}(m, f)$, as is done in [27], requires information about the signs of values of derivatives which is not as easy to obtain as in the case of values of $\Lambda_{f}(s)$. Based on the numerical data leading to Prop. 1.2, we find that, if one normalizes the odd parts of the our period polynomials to be monic, then the signs of the polynomials just to the right of 1 are - . Since 
the sign at $+\infty$ of those normalized period polynomials is + , that would imply that there are an odd (and hence positive) number of real roots $a>1$. However, we have not been able to prove theoretically that the sign just to the right of 1 is -.

The reason for calling the conjecture a "Riemann Hypothesis" is that if $z_{0}$ is a zero of $Q_{f}$, then $-1 / z_{0}$ is also a zero, as implied by the transformation

$$
Q_{f}(m,-1 / z)=(-1)^{m-1} Q_{f}(m, z) z^{2-k} .
$$

Thus, the unit circle is the natural line of symmetry in this case (and can be mapped to a "zeta polynomial" where the Riemann Hypothesis stipulates that the zeros lie on the line $\operatorname{Re}(s)=\frac{1}{2}$ in a natural framework due to Manin [26] and expounded upon by the authors of [28]). This transformation law is an implication (see Lemma 3.6 and (12)) of the cohomological structure we associate to derivatives of $L$-functions and which formed the conceptual basis for the conjecture.

As part of the evidence for the truth of this conjecture we prove the Eisenstein series case of part ii). of Conjecture 1.1 in the case of first derivative and $k \equiv 0(\bmod 4)$. This can be stated as

Theorem 1.3. Let $k \equiv 0(\bmod 4)$ and let

$$
f(\tau):=E_{k}(\tau)=-\frac{B_{k}}{2 k}+\sum_{n=1}^{\infty} \sigma_{k-1}(n) e^{2 \pi i n \tau}
$$

where $B_{n}$ denotes the $n$-th Bernoulli number. Then all zeros of

$$
P_{f}(z):=\sum_{\substack{n=1 \\
n \text { odd }}}^{k-3}\left(\begin{array}{c}
k-2 \\
n
\end{array}\right) i^{1-n} \Lambda_{f}^{\prime}(n+1) z^{k-3-n}
$$

lie on the unit circle.

\section{Motivation, background, and structure of the paper}

As mentioned above, the values of $L_{f}(s)$ themselves inside the critical strip are better understood than those of the derivatives. Important tools that have been used in their study are the period polynomial and the Eichler cohomology.

Specifically, if $f$ is a cusp form of weight $k$ for $\Gamma:=\mathrm{SL}_{2}(\mathbb{Z})$, it is possible to associate a 1-cocycle to it as follows. We consider the action $\left.\right|_{2-k}$ of $\Gamma$ on the space $\mathcal{O}$ of holomorphic maps on the upper half plane $\mathfrak{H}$, defined, for each $f: \mathfrak{H} \rightarrow \mathbb{C}$, by

$$
\left(\left.f\right|_{2-k} \gamma\right)(\tau):=f(\gamma \tau) j(\gamma, \tau)^{k-2}, \quad \tau \in \mathfrak{H}, \gamma \in \Gamma,
$$

where

$$
j\left(\left(\begin{array}{ll}
* & * \\
c & d
\end{array}\right), \tau\right):=c \tau+d
$$


We use the same notation for the restriction of this action to the space $P_{k-2}$ of polynomial functions of degree $\leq k-2$.

Then, for some $\tau_{0} \in \mathfrak{H} \cup \mathbb{Q} \cup\{\infty\}$, we assign to our cusp form $f$ a map $\sigma_{f}$ that sends $\gamma \in \Gamma$ to

$$
\int_{\tau_{0}}^{\gamma^{-1}\left(\tau_{0}\right)} f(\tau)(\tau-z)^{k-2} d \tau
$$

The map $\sigma_{f}$ is then a 1-cocycle and its cohomology class is independent of the choice of $\tau_{0}$. We call this an Eichler cocycle.

Then, taking $\tau_{0}=0$, the period polynomial of $f$ can be recovered as the value of $\sigma_{f}$ at $S:=\left(\begin{array}{cc}0 & -1 \\ 1 & 0\end{array}\right)$ :

$$
\sigma_{f}(S)=\int_{0}^{\infty} f(\tau)(\tau-z)^{k-2} d \tau .
$$

Indeed, as will be shown in greater generality in the sequel, this equals

$$
\sum_{n=0}^{k-2}\left(\begin{array}{c}
k-2 \\
n
\end{array}\right) i^{1-n} \Lambda_{f}(n+1) z^{k-2-n} .
$$

The relations satisfied by the period polynomial as a value of an Eichler cocycle (including those originating in the Hecke action compatibility of Eichler cocycles), have far-reaching consequences for the arithmetic and geometry of $f$. For instance, a fairly immediate implication of the Eichler-Shimura isomorphism applied to the period polynomial is Manin's Periods Theorem [26], which provides important information about the arithmetic nature of critical $L$-values.

The fundamental nature of the period polynomial is reflected in other aspects of its structure, for instance when viewed as a polynomial. In particular, the location of its zeros has been studied by various authors. It seems that the first case to be considered was the analogue of the period polynomial associated to Eisenstein series. Even to formulate the correct definition of period polynomials for Eisenstein series has been an important question of independent interest. The versions that will play a role in this paper are those of D. Zagier [32] and of F. Brown [3].

With the definition as in [32], M. R. Murty, C.J. Smyth and R.J.Wang [27] proved that all non-real zeros of the odd part of the period polynomial of an Eisenstein series lie on the unit circle. This is a natural line of symmetry for the period polynomials, given that they are reciprocal polynomials thanks to the functional equation for completed $L$-values, and so as explained by S. Jin, W. Ma, K. Ono, and K. Soundararajan in [20], such results can be thought of as a sort of "Riemann Hypothesis" for period polynomials. The interested reader is also referred to [28] for further results connecting such results to Manin's theory of "zeta polynomials" $Z_{f}(s)$, which are transformed versions of the period polynomials sending the unit circle to the line $\operatorname{Re}(s)=\frac{1}{2}$ and satisfying the functional equation $Z_{f}(1-s)= \pm Z_{f}(s)$. M.N. Lalín and C.J. Smyth [24] proved that all zeros of the full period polynomial of an Eisenstein series are unimodular. Analogous results have been proved for the case of cusp forms. For example, J.B. Conrey, D.W. Farmer and Ö. Imamoğlu [6] have proved that, apart 
from some "trivial" real zeros, all zeros of the odd part of the period polynomial of a cusp form lie on the unit circle. In the context of part ii). of Conjecture 1.1, these are exactly the points stated there with $a=2$. A. El-Guindy and W. Raji [14] have extended this to the full period polynomial by showing that its zeros are all unimodular. The analogues of these results for higher levels, together with very explicit approximations for the exact locations of the zeros is proved in [20].

The starting point for our extension of these results to derivatives of L-functions was the study of certain "period polynomials" attache to derivatives by D. Goldfeld and the first author. Motivated by the success of Eichler cohomology and the (classical) period polynomials, they defined analogues of the period polynomial that encode values of derivatives of $L$-functions [15, 8, 9].

Specifically, we considered the polynomial

$$
-\sum_{n=0}^{k-2}\left(\begin{array}{c}
k-2 \\
n
\end{array}\right) i^{1-n} \Lambda_{f}^{\prime}(n+1) z^{k-2-n}
$$

and computed numerically its zeros for many weights. We found that, in all cases we tested, the polynomial has its zeros on the unit circle. Likewise, the "odd part" of this polynomial seems to have zeros all on the unit circle other than 5 simple ones of a shape resembling the main results of [6].

That was surprising because, as pointed out in [23], only the first non-vanishing derivatives are normally expected to have some number-theoretic significance. However, in the polynomial in question, all integer values of derivatives inside the critical strip play an equal role in our results.

To develop a general framework for these phenomena, we had to conceptually justify our choice of the "period polynomial" encoding values of derivatives of $L$-functions. That was achieved by cohomological considerations on the basis of the classical Eichler theory and the constructions of [9].

We first (see Section 3) reinterpret the cocycles associated to values of $L$-functions of a general modular form $f$ in a way that will be consistent with our corresponding construction for derivatives of $L$-functions. Our cocycle is "canonical" in the sense that it belongs to the same cohomology class as the image of $f$ under the Eichler-Shimura isomorphism (see Proposition 3.2). It turns out that, in our interpretation, the period polynomial we associate to Eisenstein series coincides with the version of [3].

The form of the cocycles we assign to derivatives of general modular forms (Sec. 3.2) is entirely analogous to that of the cocycles we attach to values of $L$-functions. As is normally to be expected in the case of (higher) derivatives, one has to separate the "main" from the "lower order" terms. A second feature indicating that our cocycles are the "right" objects to look at is that the passage from first to second and higher derivatives is achieved via a group cohomological construction based on cup products, thus allowing for a unified treatment of all derivatives.

With these "period polynomials for derivatives of $L$-functions" in place, we turn our attention to their zeros. We prove special cases of the part ii). of Conjecture 1.1 for Eisenstein 
series. We only treat the analogues of [27] and [6], by focusing on the "odd parts" of these polynomials.

The proof of Proposition 1.2 verifying Conjecture 1.1 in a number of cases will be given in Section 5. A general discussion of numerical examples of this conjecture and its context will also be given there. We will also describe numerical experiments suggested to the authors by Don Zagier on the robustness of these conjectures. These give a measure of how far one must perturb the polynomials until the roots no longer lie on the unit circle. Finally, a discussion of possible follow-up work will be given.

\section{Acknowledgements}

The authors are grateful to K. Bringmann, F. Brown, D. Goldfeld, K. Ono, A. Straub, and J. Thorner for useful discussions, as well as to Trinity College Dublin for hosting the first author on a visit where this project arose. The authors also thank A. Babei for assistance with computational experiments and D. Zagier for many helpful suggestions and comments which improved the paper.

\section{Cocycles associated to values of $L$-functions and to values of their derivatives}

The classical Eichler-Shimura theory assigns a cocycle to a modular form $f$ in such a way that it characterizes the critical values of $L_{f}(s)$. In the case of the modular group this cocycle is determined by its value at the involution which is called "period polynomial." This was originally defined and studied for cusp forms yielding many important arithmetic results.

Zagier [32] seems to be the first one to study an extension of the period polynomial to non-cuspidal forms (although, previously, Grosswald [18] also defined similar objects from a different perspective). His definition has been generalized and modified by various authors in accordance with different perspectives. We will recall one of them in the next subsection.

Another direction in which Eichler-Shimura theory and period polynomials have been extended is to values of derivatives of $L$-functions of cusp forms. Goldfeld [15] and the first author $[8,9,10]$ have considered an approach allowing for the encoding values of derivatives into analogues of the period polynomial and which can be interpreted in the context of Eichler cohomology.

In this section, we extend the constructions of [9] and [15] to non-cuspidal modular forms. Before doing that, we consider the cocycles associated to values of $L$-functions of general modular forms in a formulation that fits the "period polynomials" we will associate to derivatives of $L$-functions in the next subsection. 


\subsection{Cocycles associated to values of $L$-functions}

This subsection outlines the known theory of cocycles associated to values of $L$-functions of general modular forms. We use a formulation that is consistent with the corresponding construction for derivatives of $L$-functions we will discuss in the next subsection.

Throughout, let $k$ be an even positive integer. We will be using the action $\left.\right|_{2-k}$ of $\Gamma$ on $\mathcal{O}$ defined by (1) and its restriction to the space $P_{k-2}$ of polynomial functions of degree $\leq k-2$.

As usual, we denote the space of $i$-cochains for $\Gamma$ with coefficients in a right $\Gamma$-module $M$ by $C^{i}(\Gamma, M)$. We will also use the formalism of "bar resolution" for the differential $d^{i}: C^{i}(\Gamma, M) \rightarrow$ $C^{i+1}(\Gamma, M)$ :

$$
\begin{aligned}
& \left(d^{i} \sigma\right)\left(g_{1}, \ldots, g_{i+1}\right):= \\
& \sigma\left(g_{2}, \ldots, g_{i+1}\right) \cdot g_{1}+\sum_{j=1}^{i}(-1)^{j} \sigma\left(g_{1}, \ldots, g_{j+1} g_{j}, \ldots, g_{i+1}\right)+(-1)^{i+1} \sigma\left(g_{1}, \ldots, g_{i}\right) .
\end{aligned}
$$

We now start the construction of the cocycles we will assign to a modular form. Let

$$
f(\tau)=\sum_{n=0}^{\infty} a_{n} e^{2 \pi i n \tau}
$$

be an element of the space $M_{k}$ of modular forms of weight $k$ for $\Gamma$. As usual, we define its $L$-function, for $\operatorname{Re}(s) \gg 1$, by

$$
L_{f}(s):=\sum_{n=1}^{\infty} \frac{a_{n}}{n^{s}}
$$

and, the "completed" $L$-function by

$$
\Lambda_{f}(s):=(2 \pi)^{-s} \Gamma(s) L_{f}(s)
$$

It is well-known (see, e.g., [19], Chapt. 7) that $\Lambda_{f}$ has a meromorphic continuation to the entire complex plane with possible (simple) poles at 0 and $k$ and that it satisfies the functional equation

$$
\Lambda_{f}(s)=i^{k} \Lambda_{f}(k-s) .
$$

It further has an integral expression:

$$
\Lambda_{f}(s)=\int_{1}^{\infty}(f(i v)-a(0)) v^{s-1} d v+i^{k} \int_{1}^{\infty}(f(i v)-a(0)) v^{k-s-1} d v-\frac{a(0)}{s}-\frac{a(0) i^{k}}{k-s}
$$

which, in the cuspidal case, reduces to the classical expression for $\Lambda_{f}$ as a Mellin transform.

Define $v_{f}^{(0)} \in C^{0}(\Gamma, \mathcal{O})$ by the Eichler integral

$$
v_{f}^{(0)}(z):=\int_{\infty}^{z}\left(f(w)-a_{0}\right)(w-z)^{k-2} d w+\frac{a_{0}}{k-1} z^{k-1} .
$$


This is well-defined because of the exponential decay of $f(w)-a_{0}$ at $\infty$. An alternative interpretation of this object, originating in geometric considerations, is the "integral at a tangential base point at infinity" of [3], (Section 4 and, in particular, (4.12)).

Set $\sigma_{f}^{(1)}:=d^{0} v_{f}^{(0)}$. The next lemma shows that this is a 1-cocycle in Eichler cohomology. It is classical but here we present a computational proof to highlight the analogy with Lemma 3.3 and because some computations are needed in the sequel. Again, a different interpretation of this is given in Lemma 5.1 of [3].

Lemma 3.1. The map $\sigma_{f}^{(1)}$ takes values in $P_{k-2}$. In particular, it gives a 1-cocycle in $P_{k-2}$.

Proof. We first note that $z^{k-1} /(k-1)=\int_{0}^{z}(w-z)^{k-2} d w$. Therefore, the value of $\sigma_{f}^{(1)}(\gamma)=$ $\left.v_{f}^{(0)}\right|_{2-k}(\gamma-1)$ at $z$ equals

$$
\begin{aligned}
& \left.\left(\int_{i}^{z} f(w)(w-z)^{k-2} d w\right)\right|_{2-k}(\gamma-1)-\left.a_{0}\left(\int_{i}^{z}(w-z)^{k-2} d w\right)\right|_{2-k}(\gamma-1) \\
& +\left.\int_{\infty}^{i}\left(f(w)-a_{0}\right)(w-z)^{k-2} d w\right|_{2-k}(\gamma-1)+\left.a_{0}\left(\int_{0}^{z}(w-z)^{k-2} d w\right)\right|_{2-k}(\gamma-1) .
\end{aligned}
$$

The third term is clearly in $P_{k-2}$. On the first, we use the elementary identity

$$
(w-\gamma z) j(\gamma, z)=\left(\gamma^{-1} w-z\right) j\left(\gamma^{-1}, w\right)
$$

and the modular invariance of $f$ to get

$$
\begin{aligned}
& \int_{i}^{\gamma z} f(w)(w-\gamma z)^{k-2} j(\gamma, z)^{k-2} d w-\int_{i}^{z} f(w)(w-z)^{k-2} d w \\
& =\int_{i}^{\gamma z} f\left(\gamma^{-1} w\right)\left(\gamma^{-1} w-z\right)^{k-2} d\left(\gamma^{-1} w\right)-\int_{i}^{z} f(w)(w-z)^{k-2} d w=\int_{\gamma^{-1} i}^{i} f(w)(w-z)^{k-2} d w,
\end{aligned}
$$

which is in $P_{k-2}$. The remaining two terms of (5) combine to $\left.a_{0}\left(\int_{0}^{i}(w-z)^{k-2} d w\right)\right|_{k-2}(\gamma-1)$, which is also in $P_{k-2}$.

Further, since $\sigma_{f}^{(1)}$ is given as the differential of a 1-cochain, it will satisfy the 1-cocycle relation.

The next proposition, on the one hand shows that $\sigma_{f}^{(1)}$ is canonical in the sense that it belongs to the cohomology class associated to $f$ under the Eichler Shimura isomorphism. On the other, it makes explicit the relation of $\sigma_{f}^{(1)}$ with the critical $L$-values of $f$.

Specifically, we will first prove that $\sigma_{f}^{(1)}$ belongs to the cohomology class of $f$ under the Eichler Shimura isomorphism

$$
\phi: \overline{S_{k}} \oplus M_{k} \stackrel{\sim}{\rightarrow} H^{1}\left(\Gamma, P_{k-2}\right) .
$$


The isomorphism $\phi$ is induced by the assignment of $f \in M_{k}$ to the map $\phi(f)$ such that

$$
\phi(f)(\gamma)=\int_{\gamma^{-1} i}^{i} f(w)(w-z)^{k-2} d w \quad \text { for } \gamma \in \Gamma
$$

Secondly we will show that the value of the cocycle $\sigma_{f}^{(1)}$ at $S$ encodes the critical $L$-values of $f$. When $f$ is cuspidal, $\sigma_{f}^{(1)}(S)$ is essentially the classical period polynomial, whereas, for $f=E_{k}$, it is the period polynomial $\mathrm{p}\left(E_{k}\right)$ of [3], which plays an important role there. From this viewpoint, (8) can be thought of as a formula for the full Eichler cocycle whose specialization at $S$ is Brown's period polynomial of an Eisenstein series.

Proposition 3.2. If $f \in M_{k}$, then the following are true.

i). The 1-cocycle $\sigma_{f}^{(1)}$ is a representative of the cohomology class of $\phi(f)$.

ii). For each $z \in \mathbb{C}$, we have

$$
\sigma_{f}^{(1)}(S)=-i \sum_{j=0}^{k-2}\left(\begin{array}{c}
k-2 \\
j
\end{array}\right)(i z)^{j} \Lambda_{f}(j+1)
$$

Proof. We begin with the proof of i). Using (5) and (7), we see that

$$
\begin{aligned}
\sigma_{f}^{(1)}(\gamma) & =\int_{\gamma^{-1} i}^{i} f(w)(w-z)^{k-2} d w \\
& +\left.\left(\int_{\infty}^{i}\left(f(w)-a_{0}\right)(w-z)^{k-2} d w+a_{0} \int_{0}^{i}(w-z)^{k-2} d w\right)\right|_{2-k}(\gamma-1) .
\end{aligned}
$$

Since the part inside the parentheses is in $P_{k-2}$, the second row of (8) is a coboundary and thus $\sigma_{f}^{(1)}$ differs from $\phi(f)$ by a coboundary.

We now turn to the proof of ii). Setting $\gamma=S$ in (8), we see that $\sigma_{f}^{(1)}(S)$ equals

$$
\int_{\infty}^{i}\left(f(w)-a_{0}\right)\left((w z+1)^{k-2}-(w-z)^{k-2}\right) d w+\left.\frac{a_{0}}{k-1}\left((i-z)^{k-1}-(-z)^{k-1}\right)\right|_{2-k}(S-1) .
$$

The binomial expansion and (4) imply the identity after an elementary calculation.

\subsection{Cocycles associated to values of derivatives of $L$-functions}

In this section, we will associate 2-cocycles to values of derivatives of L-functions. It should be stressed that this is purely a theory of cocycles not one of cohomology. The intrinsic reason is that, if $j \geq 2$, then $H^{j}\left(\mathrm{SL}_{2}(\mathbb{Z}), M\right)=0$ for each $\mathbb{C}$-vector space $M$ (see, e.g. Lemma 7.2 of $[12])$. 
We maintain the notation of the previous section. We further let $u(\tau)$ be a holomorphic function on $\mathfrak{H}$ with polynomial growth as $\tau$ approaches $q \in \mathbb{Q} \cup\{\infty\}$. We will choose a specific $u$ later on in order to prove Prop. 3.5.

Define the cochain $v_{f}^{(1)} \in C^{1}(\Gamma, \mathcal{O})$ by

$$
\begin{aligned}
v_{f}^{(1)}(\gamma)(z) & :=\int_{\infty}^{z}\left(f(w)-a_{0}\right)(w-z)^{k-2}(u(\gamma w)-u(w)) d w \\
& +a_{0} \int_{i}^{z}(w-z)^{k-2}(u(\gamma w)-u(w)) d w
\end{aligned}
$$

Set $\sigma_{f}^{(2)}:=d^{1} v_{f}^{(1)}$. It is a 2-cocycle in Eichler cohomology:

Lemma 3.3. The map $\sigma_{f}^{(2)}$ takes values in $P_{k-2}$ and thus gives a 2 -cocycle in $P_{k-2}$.

Proof. The value of $v_{f}^{(1)}\left(\gamma_{2}\right)(z)$ at $z$ equals

$$
\int_{i}^{z} f(w)(w-z)^{k-2}\left(u\left(\gamma_{2} w\right)-u(w)\right) d w+\int_{\infty}^{i}\left(f(w)-a_{0}\right)(w-z)^{k-2}\left(u\left(\gamma_{2} w\right)-u(w)\right) d w .
$$

The image of the second term under the differential $d^{1}$ (see (2)) is clearly in $P_{k-2}$. The image of the first term equals

$$
\begin{aligned}
& \int_{i}^{\gamma_{1} z} f(w)\left(w-\gamma_{1} z\right)^{k-2} j\left(\gamma_{1}, z\right)^{k-2}\left(u\left(\gamma_{2} w\right)-u(w)\right) d w \\
& -\int_{i}^{z} f(w)(w-z)^{k-2}\left(u\left(\gamma_{2} \gamma_{1} w\right)-u\left(\gamma_{1} w\right)\right) d w .
\end{aligned}
$$

With (6) and the modularity of $f$, we deduce that this equals

$$
-\int_{i}^{\gamma_{1}^{-1} i} f(w)(w-z)^{k-2}\left(u\left(\gamma_{2} \gamma_{1} w\right)-u\left(\gamma_{1} w\right)\right) d w
$$

which is in $P_{k-2}$.

The next proposition shows that when $f$ is cuspidal, $\sigma_{f}^{(2)}$ coincides with the cocycle associated to derivatives of $L$-functions in [9].

Proposition 3.4. Let $f$ be a cusp form of weight $k$ for $\Gamma$. Then

$$
\begin{aligned}
\sigma_{f}^{(2)}\left(\gamma_{1}, \gamma_{2}\right) & =\int_{\gamma_{1}^{-1} \infty}^{\infty} f(w)(w-z)^{k-2}\left(u\left(\gamma_{2} w\right)-u(w)\right) d w \\
& =\left.\int_{\infty}^{\gamma_{1} \infty} f(w)(w-z)^{k-2}\left(u\left(\gamma_{2} w\right)-u(w)\right) d w\right|_{2-k} \gamma_{1}
\end{aligned}
$$


Proof. When $f$ is cuspidal, then

$$
v_{f}^{(1)}(\gamma)(z)=\int_{\infty}^{z} f(w)(w-z)^{k-2}(u(\gamma w)-u(w)) d w
$$

and this implies that

$$
\begin{aligned}
\sigma_{f}^{(2)}\left(\gamma_{1}, \gamma_{2}\right) & =\int_{\infty}^{\gamma_{1} z} f(w)\left(w-\gamma_{1} z\right)^{k-2} j\left(\gamma_{1}, z\right)^{k-2}\left(u\left(\gamma_{2} w\right)-u(w)\right) d w \\
& -\int_{\infty}^{z} f(w)(w-z)^{k-2}\left(u\left(\gamma_{2} \gamma_{1} w\right)-u\left(\gamma_{1} w\right)\right) d w
\end{aligned}
$$

Equation (6) and the modularity of $f$ imply that, after the change of variables $\gamma^{-1} w \rightarrow w$, the first integral equals

$$
\int_{\gamma_{1}^{-1} \infty}^{z} f(w)(w-z)^{k-2}\left(u\left(\gamma_{2} \gamma_{1} w\right)-u\left(\gamma_{1} w\right)\right) d w
$$

which gives the first equality. The second follows from a change of variables and (6).

We now specialize $u(\tau)$ to $2 \log (\eta(\tau))$ where $\eta(\tau)$ is the Dedekind eta function and $\log$ takes the principal branch so that

$$
\log (\eta(\tau))=\frac{\pi i \tau}{12}-\sum_{n=1}^{\infty} \sum_{m=1}^{\infty} \frac{1}{m} e^{2 \pi i n m \tau}
$$

for each $\tau \in \mathfrak{H}$. This function satisfies (see, e.g. $\S 3$ of [30] for a proof):

$$
\begin{aligned}
& \log (\eta(\tau+1))=\log (\eta(\tau))+\pi i / 12 \\
& \log (\eta(-1 / \tau))=u(\tau)+\frac{1}{2} \log (\tau / i)
\end{aligned}
$$

With this definition of $\sigma_{f}^{(2)}$, we will show that, up to a simple multiple of a fixed polynomial, $\sigma_{f}^{(2)}$ encodes the values of derivatives of the $L$-function of $f$ inside the critical strip just as the cuspidal analogue in [9] did.

Proposition 3.5. Set

$$
P(z)=\sum_{n=0}^{k-2}\left(\begin{array}{c}
k-2 \\
n
\end{array}\right) \frac{i^{1-n}}{(n+1)^{2}} z^{k-2-n}
$$

Then

$$
\sigma_{f}^{(2)}(S, S)=-\sum_{n=0}^{k-2}\left(\begin{array}{c}
k-2 \\
n
\end{array}\right) i^{1-n} \Lambda_{f}^{\prime}(n+1) z^{k-2-n}+a(0)\left(\left.P\right|_{2-k}(1+S)\right)(z) .
$$


Proof. Upon differentiation of (4), we obtain

$$
\Lambda_{f}^{\prime}(s)=\int_{1}^{\infty}(f(i v)-a(0)) v^{s-1} \log (v) d v-i^{k} \int_{1}^{\infty}(f(i v)-a(0)) v^{k-s-1} \log (v) d v+\frac{a(0)}{s^{2}}-\frac{a(0) i^{k}}{(k-s)^{2}} .
$$

Equation (9) gives $\log (v)=u(\operatorname{Siv})-u(i v)$, and so

$$
\begin{aligned}
& \sum_{n=0}^{k-2}\left(\begin{array}{c}
k-2 \\
n
\end{array}\right) i^{1-n} \Lambda_{f}^{\prime}(n+1) z^{k-2-n}= \\
& \sum_{n=0}^{k-2}\left(\begin{array}{c}
k-2 \\
n
\end{array}\right) i^{1-n} z^{k-2-n}\left(i^{-n-1} \int_{i}^{i \infty}(f(w)-a(0)) w^{n}(u(S w)-u(w)) d w\right. \\
& \left.-i^{n+1} \int_{i}^{i \infty}(f(w)-a(0)) w^{k-n-2}(u(S w)-u(w)) d w-a(0)\left(\frac{i^{k}}{(k-n-1)^{2}}-\frac{1}{(n+1)^{2}}\right)\right) \\
& =\int_{i}^{i \infty}(f(w)-a(0))(z-w)^{k-2}(u(S w)-u(w)) d w \\
& +\int_{i}^{i \infty}(f(w)-a(0))(z w+1)^{k-2}(u(S w)-u(w)) d w \\
& +a(0)\left(P(z)+P(-1 / z) z^{k-2}\right)=-\sigma_{f}^{(2)}(S, S)+a(0)\left(\left.P\right|_{2-k}(1+S)\right)(z) .
\end{aligned}
$$

Remark. It is possible to eliminate $P$ from the statement Proposition 3.5 by modifying the definition of $v_{f}^{(1)}$ to match, in some respects, even more perfectly the $v_{f}^{(0)}$ we assigned to the values of $L$-functions in the previous subsection. However, the formula would become more complicated without an obvious benefit.

\subsection{Higher derivatives}

We can now extend the construction above to account for all derivatives of $L$-functions. For the reason explained in the preamble of Section 3.2, this is not a cohomological construction but one of cocycles.

It will be convenient to use the group cohomology formalism of cup products. We consider the cup product map

$$
\cup: C^{1}(\Gamma, \mathcal{O}) \otimes C^{m}(\Gamma, \mathcal{O}) \rightarrow C^{m+1}(\Gamma, \mathcal{O})
$$

given by

$$
\left(\phi_{1} \cup \phi_{2}\right)\left(\gamma_{1}, \gamma_{2}, \ldots, \gamma_{m+1}\right):=\phi_{1}\left(\gamma_{1}\right)\left(\left.\phi\left(\gamma_{2}, \ldots, \gamma_{m+1}\right)\right|_{0} \gamma_{1}\right) \text {. }
$$

For $\phi_{i} \in C^{1}(\Gamma, \mathcal{O})$, we consider the iterated product:

$$
\phi_{1} \cup \cdots \cup \phi_{n}:=\phi_{1} \cup\left(\phi_{2} \cup\left(\ldots\left(\phi_{n-1} \cup \phi_{n}\right) \ldots\right)\right) \in C^{n}(\Gamma, \mathcal{O}) .
$$

An important property is that cup products of cocycles are cocycles. 
With this notation, we set, for $n \in \mathbb{N}$,

$$
V_{n}:=v \cup v \cup \cdots \cup v \quad(n \text { times })
$$

where $v$ is 1-cocycle given by $\left.\gamma \rightarrow u\right|_{0}(\gamma-1)$ (with $u$ as in the last subsection). As mentioned above, this will be a $n$-cocycle.

Let $v_{f}^{(n)} \in C^{n}(\Gamma, \mathcal{O})$ be given by

$$
\begin{aligned}
v_{f}^{(n)}\left(\gamma_{1}, \ldots, \gamma_{n}\right)(z) & =\int_{\infty}^{z}\left(f(w)-a_{0}\right)(w-z)^{k-2} V_{n}\left(\gamma_{1}, \ldots, \gamma_{n}\right)(w) d w \\
& +a_{0} \int_{i}^{z}(w-z)^{k-2} V_{n}\left(\gamma_{1}, \ldots, \gamma_{n}\right)(w) d w
\end{aligned}
$$

Setting $\sigma_{f}^{(n+1)}:=d^{n} v_{f}^{(n)}$, we arrive at the following analogue of Lemma 3.3 for higher cocycles.

Lemma 3.6. The map $\sigma_{f}^{(n+1)}$ takes values in $P_{k-2}$ and thus gives an $(n+1)$-cocycle in $P_{k-2}$. Proof. The value of $v_{f}^{(n)}\left(\gamma_{2}, \ldots, \gamma_{n+1}\right)$ at $z$ equals

$$
\int_{i}^{z} f(w)(w-z)^{k-2} V_{n}\left(\gamma_{2}, \ldots, \gamma_{n+1}\right)(w) d w+\int_{\infty}^{i}\left(f(w)-a_{0}\right)(w-z)^{k-2} V_{n}\left(\gamma_{2}, \ldots, \gamma_{n+1}\right)(w) d w
$$

The image of the second term under the differential $d^{n}$ is clearly in $P_{k-2}$. The image of the first term equals

$$
\begin{gathered}
\int_{i}^{\gamma_{1} z} f(w)\left(w-\gamma_{1} z\right)^{k-2} j\left(\gamma_{1}, z\right)^{k-2} V_{n}\left(\gamma_{2}, \ldots, \gamma_{n+1}\right)(w) d w+\int_{i}^{z} f(w)(w-z)^{k-2} \times \\
\left\{\sum_{j=1}^{n}(-1)^{j} V_{n}\left(\gamma_{1}, \ldots, \gamma_{j+1} \gamma_{j}, \ldots, \gamma_{n+1}\right)(w)+(-1)^{n+1} V_{n}\left(\gamma_{1}, \ldots, \gamma_{n}\right)(w)\right\} d w .
\end{gathered}
$$

Since $V_{n}$ is a $n$-cocycle in terms of the action of $\left.\right|_{0}$ on $\mathcal{O}$, the part inside the curly brackets equals

$$
-V_{n}\left(\gamma_{2}, \ldots, \gamma_{n+1}\right)\left(\gamma_{1} w\right) .
$$

On the other hand, (6) and the modularity of $f$ imply that the first integral in (11) equals

$$
\int_{\gamma_{1}^{-1} i}^{z} f(w)(w-z)^{k-2} V_{n}\left(\gamma_{2}, \ldots, \gamma_{n+1}\right)\left(\gamma_{1} w\right) d w
$$

Thus, (11) equals

$$
\int_{\gamma_{1}^{-1} i}^{i} f(w)(w-z)^{k-2} V_{n}\left(\gamma_{2}, \ldots, \gamma_{n+1}\right)\left(\gamma_{1} w\right) d w
$$

which is in $P_{k-2}$. 
Finally, we describe the relation with the higher derivatives of $L$-functions in the critical strip.

Proposition 3.7. For each $m \in \mathbb{N}$, set

$$
P(z)=\sum_{n=0}^{k-2}\left(\begin{array}{c}
k-2 \\
n
\end{array}\right) \frac{i^{1-n}}{(-n-1)^{m+1}} z^{k-2-n}
$$

Then

$(-1)^{m} \sigma_{f}^{(m+1)}(S, \ldots S)=\sum_{n=0}^{k-2}\left(\begin{array}{c}k-2 \\ n\end{array}\right) i^{1-n} \Lambda_{f}^{(m)}(n+1) z^{k-2-n}-a(0) m !\left(\left.P\right|_{2-k}\left(1+(-1)^{m+1} S\right)\right)(z)$,

where $\sigma_{f}^{(m+1)}$ has $m+1$ arguments.

Proof. As before, we have

$$
\begin{aligned}
\Lambda_{f}^{(m)}(s) & =\int_{1}^{\infty}(f(i y)-a(0)) y^{s-1} \log ^{m}(y) d y \\
& +(-1)^{m} i^{k} \int_{1}^{\infty}(f(i y)-a(0)) y^{k-s-1} \log ^{m}(y) d y+\frac{a(0) m !}{(-s)^{m+1}}+\frac{a(0) m ! i^{k}}{(s-k)^{m+1}} .
\end{aligned}
$$

We deduce the result mutatis mutandis by working as in Proposition 3.5.

\section{Zeros of "period polynomials"}

In this section we will prove that, in the special case that $f$ is an Eisenstein series, the zeros of the "odd part" of the "period polynomials" we have attached to values of derivatives of $L$-functions lie on the unit circle.

To highlight more clearly the key ideas, we study on its own the case of first derivative and then show how this can be generalized to higher derivatives.

\subsection{The case of the first derivative}

We will now prove the analogue of Theorem 5.1 of [27] for first derivatives. For simplicity, we focus on the case of weight $k \equiv 0(\bmod 4)$. Consider the piece of $\sigma_{f}^{(2)}(S, S)$ in Proposition 3.5 that contains the first derivatives of $L$-functions, i.e.,

$$
-\sum_{n=0}^{k-2}\left(\begin{array}{c}
k-2 \\
n
\end{array}\right) i^{1-n} \Lambda_{f}^{\prime}(n+1) z^{k-2-n}
$$

We will study the part of this polynomial that captures the values of $\Lambda_{f}^{\prime}$ at even arguments

$$
P_{f}(z):=\sum_{\substack{n=1 \\
n \text { odd }}}^{k-3}\left(\begin{array}{c}
k-2 \\
n
\end{array}\right) i^{1-n} \Lambda_{f}^{\prime}(n+1) z^{k-3-n}
$$


(For simplicity, we factor out a $-z$ from (13) because it only affects trivially the zeros of the polynomial.)

Theorem 4.1. Let $k \equiv 0(\bmod 4)$ and let

$$
f(\tau):=E_{k}(\tau)=-\frac{B_{k}}{2 k}+\sum_{n=1}^{\infty} \sigma_{k-1}(n) e^{2 \pi i n \tau} .
$$

Then all zeros of $P_{f}(z)$ lie on the unit circle.

Proof. By (3), our polynomial can be expressed by

$$
-\sum_{\substack{n=1 \\
n \text { odd }}}^{k-3}\left(\begin{array}{c}
k-2 \\
n
\end{array}\right) i^{n-1} \Lambda_{f}^{\prime}(n+1) z^{n-1}=-\sum_{n=0}^{k / 2-2}\left(\begin{array}{c}
k-2 \\
2 n+1
\end{array}\right) i^{2 n} \Lambda_{f}^{\prime}(2 n+2) z^{2 n}
$$

The assertion of the theorem is then equivalent to the statement that the zeros of

$$
-\sum_{n=0}^{k / 2-2}\left(\begin{array}{c}
k-2 \\
2 n+1
\end{array}\right) \Lambda_{f}^{\prime}(2 n+2)(-z)^{n}
$$

are all on the unit circle.

Since, by $(3), \Lambda_{f}^{\prime}(k / 2)=0$, we have that

$$
\begin{aligned}
& -\sum_{n=0}^{k / 2-2}\left(\begin{array}{c}
k-2 \\
2 n+1
\end{array}\right) \Lambda_{f}^{\prime}(2 n+2)(-z)^{n} \\
= & -\sum_{n=0}^{k / 4-2}\left(\begin{array}{c}
k-2 \\
2 n+1
\end{array}\right) \Lambda_{f}^{\prime}(2 n+2)(-z)^{n}-\sum_{n=k / 4}^{k / 2-2}\left(\begin{array}{c}
k-2 \\
2 n+1
\end{array}\right) \Lambda_{f}^{\prime}(2 n+2)(-z)^{n} \\
= & -\sum_{n=0}^{k / 4-2}\left(\begin{array}{c}
k-2 \\
2 n+1
\end{array}\right) \Lambda_{f}^{\prime}(2 n+2)(-z)^{n}+q_{f}(z)=-q_{f}(1 / z) z^{k / 2-2}+q_{f}(z)
\end{aligned}
$$

where

$$
q_{f}(z):=-\sum_{n=k / 4}^{k / 2-2}\left(\begin{array}{c}
k-2 \\
2 n+1
\end{array}\right) \Lambda_{f}^{\prime}(2 n+2)(-z)^{n} .
$$

(At the last step we made the change of variables $n \rightarrow k / 2-2-n$ and used (3) and that $k / 2-2$ is even.) The theory of "self-inversive" polynomials (see, for instance Th. 2.2 of [14]) implies that it suffices to show that all zeroes of $q_{f}(z)$ are in $|z| \leq 1$.

To show this, we first re-write $\Lambda_{f}^{\prime}(2 n+2)$. It is well-known that the $L$-function of $E_{k}$ equals $\zeta(s) \zeta(s-k+1)$ and, thus, together with the functional equation for $\zeta(s)$ (in the form 
with the sine function) we have

$$
\begin{aligned}
\Lambda_{f}(s) & =\frac{\Gamma(s)}{(2 \pi)^{s}} \zeta(s) \frac{2^{s-k+1}}{\pi^{k-s}} \Gamma(k-s) \sin \left(\frac{\pi}{2}(s-k+1)\right) \zeta(k-s) \\
& =2(2 \pi)^{-k} \cos \left(\frac{\pi s}{2}\right) \Gamma(s) \Gamma(k-s) \zeta(s) \zeta(k-s) .
\end{aligned}
$$

Therefore,

$$
\Lambda_{f}^{\prime}(s)=\Lambda_{f}(s)\left(\frac{-\pi \sin \left(\frac{\pi s}{2}\right)}{2 \cos \left(\frac{\pi s}{2}\right)}+\frac{\Gamma^{\prime}(s)}{\Gamma(s)}-\frac{\Gamma^{\prime}(k-s)}{\Gamma(k-s)}+\frac{\zeta^{\prime}(s)}{\zeta(s)}-\frac{\zeta^{\prime}(k-s)}{\zeta(k-s)}\right),
$$

and thus for $n \geq 0$ we see that $\Lambda_{f}^{\prime}(2 n+2)$ equals

$$
\begin{aligned}
& \frac{2}{(2 \pi)^{k}}(2 n+1) !(k-2 n-3) ! \cos (\pi(n+1)) \zeta(2 n+2) \zeta(k-2 n-2) \\
& \times\left(H_{2 n+1}-H_{k-2 n-3}+\frac{\zeta^{\prime}(2 n+2)}{\zeta(2 n+2)}-\frac{\zeta^{\prime}(k-2 n-2)}{\zeta(k-2 n-2)}\right) .
\end{aligned}
$$

Here $H_{n}:=\sum_{l=1}^{n} 1 / l$, and we used (5.4.14) of [29].

Thus,

$$
\begin{aligned}
q_{f}(z) & =\frac{-2(k-2) !}{(2 \pi)^{k}} \sum_{n=k / 4}^{k / 2-2} \zeta(2 n+2) \zeta(k-2 n-2)(-1)^{n+1} \\
& \times\left(H_{2 n+1}-H_{k-2 n-3}+\frac{\zeta^{\prime}(2 n+2)}{\zeta(2 n+2)}-\frac{\zeta^{\prime}(k-2 n-2)}{\zeta(k-2 n-2)}\right)(-z)^{n} .
\end{aligned}
$$

It is now clear that $H_{n} \leq H_{m}$ when $n \leq m$, and that $\zeta^{\prime}(s) / \zeta(s)$ is negative and increasing for $s>1$, which follows from the well-known Dirichlet series expansion

$$
\frac{\zeta^{\prime}(s)}{\zeta(s)}=-\sum_{n=1}^{\infty} \frac{\Lambda(n)}{n^{s}}
$$

where $\Lambda(n)$ is the von Mangoldt function. Therefore, for $n, m$ with $k / 4 \leq n \leq m \leq k / 2-2$, we have

$$
\begin{aligned}
0 & \leq H_{2 n+1}-H_{k-2 n-3}+\frac{\zeta^{\prime}(2 n+2)}{\zeta(2 n+2)}-\frac{\zeta^{\prime}(k-2 n-2)}{\zeta(k-2 n-2)} \\
& \leq H_{2 m+1}-H_{k-2 m-3}+\frac{\zeta^{\prime}(2 m+2)}{\zeta(2 m+2)}-\frac{\zeta^{\prime}(k-2 m-2)}{\zeta(k-2 m-2)} .
\end{aligned}
$$

It further follows that $f(x):=\zeta(2 x+2) \zeta(k-2 x-2)$ is increasing in $[k / 4-1, k / 2-2]$ because then

$$
f^{\prime}(x)=2 f(x)\left(\frac{\zeta^{\prime}(2 x+2)}{\zeta(2 x+2)}-\frac{\zeta^{\prime}(k-2 x-2)}{\zeta(k-2 x-2)}\right)>0 .
$$

Therefore the coefficients of $z^{n}$ in $q_{f}(z)$ form a non-negative and increasing sequence and thus, the Eneström-Kakeya Theorem [13, 21] implies that the zeros of $q_{f}(z)$ are all in $|z| \leq 1$. Therefore, Theorem 2.2 of [14] implies the theorem. 
The conclusion of this theorem differs slightly from the analogous results in [27] because the latter prove that the odd part of the period polynomial has some additional "trivial" zeros. The explanation of this apparent lack of analogy is that our construction of $P_{f}$ is based on Brown's period polynomial for Eisenstein series $\left(r_{E_{k}}\right)$, whereas [27] studies Zagier's period polynomial $\left(\tilde{r}_{E_{k}}\right)$. We can then complete the analogy by proving the counterpart, for $r_{E_{k}}$, of the result of [27]. This, at the same time, answers a question of Berndt and Straub in [1] (see the question about the odd part of $p_{m}$ in Remark 7.4). For convenience we restrict to the case that $4 \mid k$.

Proposition 4.2. For each $k \equiv 0 \bmod 4$, all zeros of

$$
\sum_{j=0}^{k / 2-2} \frac{B_{2 j+2}}{(2 j+2) !} \frac{B_{k-2 j-2}}{(k-2 j-2) !} z^{2 j}
$$

are on the unit circle.

Proof. It is clear that the statement is equivalent to the unimodularity of the zeros of

$$
\sum_{j=1}^{k / 2-1} \frac{B_{2 j}}{(2 j) !} \frac{B_{k-2 j}}{(k-2 j) !} z^{j-1}
$$

and that this polynomial can be written as $\ell_{f}(z)+\ell_{f}(1 / z) z^{k / 2-2}$ where

$$
\ell_{f}(z)=\sum_{j=k / 4+1}^{k / 2-1} \frac{B_{2 j}}{(2 j) !} \frac{B_{k-2 j}}{(k-2 j) !} z^{j-1}+\frac{1}{2} \frac{B_{k / 2}^{2}}{(k / 2) !^{2}} z^{k / 4-1} .
$$

As in the proof of the previous theorem, it is enough to prove that all zeros of $\ell_{f}(z)$ are in $|z| \leq 1$. To deduce that, we recall the classical identity:

$$
\frac{B_{2 n}}{(2 n) !}=\frac{(-1)^{n-1} 2 \zeta(2 n)}{(2 \pi)^{2 n}}
$$

Then

$$
\ell_{f}(z)=4(2 \pi)^{-k}\left(\sum_{j=k / 4+1}^{k / 2-1} \zeta(2 j) \zeta(k-2 j) z^{j-1}+\frac{1}{2} \zeta(k / 2)^{2} z^{k / 4-1}\right) .
$$

Since, as shown in the proof of Th. $4.1, \zeta(2 x+2) \zeta(k-2 x-2)$ is increasing for $x \in[k / 4-$ $1, k / 2-2]$, Eneström-Kakeya's theorem implies the result.

\subsection{Zeros of polynomials associated to higher derivatives}

For $j=0,1, \ldots$ set

$$
\Psi_{j+1}(s):=\frac{\partial^{j}}{\partial s^{j}}\left(\frac{\Gamma^{\prime}(s)}{\Gamma(s)}-\frac{\Gamma^{\prime}(k-s)}{\Gamma(k-s)}\right)
$$


and

$$
Z_{j+1}(s):=\frac{\partial^{j}}{\partial s^{j}}\left(\frac{\zeta^{\prime}(s)}{\zeta(s)}-\frac{\zeta^{\prime}(k-s)}{\zeta(k-s)}\right) .
$$

We further set $f(s):=-\frac{\pi}{2} \cdot \tan \left(\frac{\pi s}{2}\right)$ and we note that for even integers $s$, and for $j \in \mathbb{N}$,

$$
f^{(j)}(s)= \begin{cases}0 & \text { if } j \in 2 \mathbb{N}, \\ \frac{(-1)^{\frac{j+1}{2}} B_{j+1}\left(2^{j+1}-1\right) \pi^{j+1}}{(j+1)} & \text { if } j \in 2 \mathbb{N}+1,\end{cases}
$$

where $B_{n}$ denotes the $n$-th Bernoulli number. Since it does not depend on $s \in 2 \mathbb{Z}$, denote this constant by $b_{j+1}$ (so that $b_{1}=0, b_{2}=-\pi / 2, b_{3}=0, \ldots$ ).

An iterated application of the Leibniz rule to (15) implies, by induction, that

$$
\Lambda_{f}^{(m+1)}(s)=\Lambda_{f}(s) \sum^{*} c_{i_{1}, \ldots, j_{1}, \ldots, k_{1}, \ldots, n_{i_{1}}, \ldots, n_{j_{1}} \ldots} b_{i_{1}}^{n_{i_{1}}} \ldots \Psi_{j_{1}}^{n_{j_{1}}}(s) \cdots Z_{k_{1}}^{n_{k_{1}}}(s) \cdots
$$

for some $c_{i_{1}, \ldots, j_{1}, \ldots, k_{1}, \ldots, n_{i_{1}}, \ldots, n_{j_{1}} \ldots} \geq 0$, where the star indicates that the sum ranges over all positive $i_{1}, \ldots, j_{1}, \ldots, k_{1}, \ldots, n_{i_{1}}, \ldots, n_{j_{1}}, \ldots$ such that

$$
i_{1} n_{i_{1}}+\cdots+j_{1} n_{j_{1}}+\cdots+k_{1} n_{k_{1}}+\cdots=m+1 .
$$

We consider the part of the sum in the right hand side of (18) that does not include the lower order terms arising from the constants $b_{j}$ :

$$
\sum^{*} c_{j_{1}, \ldots, k_{1}, \ldots, n_{j_{1}}, \ldots, n_{k_{1}} \ldots} \Psi_{j_{1}}^{n_{j_{1}}}(s) \ldots Z_{k_{1}}^{n_{k_{1}}}(s) \ldots
$$

Working as above, (14) shows that the $(m+1)$-st derivative of

$$
\widetilde{\Lambda}_{f}(s):=\frac{\Lambda_{f}(s)}{\cos \left(\frac{\pi s}{2}\right)}
$$

equals that part, i.e.,

$$
\widetilde{\Lambda}_{f}^{(m+1)}(s)=\widetilde{\Lambda}_{f}(s) \sum^{*} c_{j_{1}, \ldots, k_{1}, \ldots, n_{j_{1}}, \ldots, n_{k_{1}} \ldots} \Psi_{j_{1}}^{n_{j_{1}}}(s) \ldots Z_{k_{1}}^{n_{k_{1}}}(s) \ldots
$$

In view of this, set

$$
P_{f}^{m}(z):=\sum_{\substack{n=1 \\
n \text { odd }}}^{k-3}\left(\begin{array}{c}
k-2 \\
n
\end{array}\right) i^{1-n} \widetilde{\Lambda}_{f}^{(m)}(n+1) z^{k-3-n} .
$$

Then we have

Theorem 4.3. Let $k \equiv 0(\bmod 4)$ and $m \geq 1$. Then all zeros of $P_{f}^{m}(z)$ lie on the unit circle. 
Proof. Equation (14) implies that

$$
\widetilde{\Lambda}_{f}(s)=\widetilde{\Lambda}_{f}(k-s)
$$

and therefore $\widetilde{\Lambda}_{f}^{(m)}(s)=(-1)^{m} \widetilde{\Lambda}_{f}^{(m)}(k-s)$. Thus the claim is equivalent to the unimodularity of the zeros of

$$
\sum_{n=0}^{k / 2-2}\left(\begin{array}{c}
k-2 \\
2 n+1
\end{array}\right) \widetilde{\Lambda}_{f}^{(m)}(2 n+2) z^{n}
$$

As in the proof of Th. 4.1, this polynomial can be expressed as

$$
q_{f}^{m}(z)+(-1)^{m} q_{f}^{m}(1 / z) z^{k / 2-2}
$$

where

$$
q_{f}^{m}(z):=\sum_{n=k / 4-1}^{k / 2-2} \delta_{n}\left(\begin{array}{c}
k-2 \\
2 n+1
\end{array}\right) \widetilde{\Lambda}_{f}^{(m)}(2 n+2) z^{n}
$$

where $\delta_{n}=1 / 2$ if $n=k / 4-1$, and 1 otherwise. With Th. 2.2 of [14], for the proof of unimodularity of the zeroes $P_{f}^{m}(z)$ it suffices to show that the zeros of $q_{f}^{m}$ are in $|z| \leq 1$.

To prove this we will use the following

Lemma 4.4. For each $j=1,2, \ldots$, the functions $\Psi_{j}(s)$ and $Z_{j}(s)$ are non-negative and increasing in $[k / 2, k-2]$.

Proof. Eq. (5.15.1) of [29] implies

$$
\Psi_{j+1}(s)=j ! \sum_{r \geq 0}\left(\frac{(-1)^{j+1}}{(s+r)^{j+1}}+\frac{1}{(r+k-s)^{j+1}}\right) .
$$

It is enough to show that each term in this series is non-negative: This is obvious for $s \in$ $[k / 2, k-2]$ when $j$ is odd. If $j$ is even, we have, for each $r \geq 0$,

$$
s+r>r+k-s>0 \quad \text { and thus } \frac{1}{(r+k-s)^{j+1}}-\frac{1}{(s+r)^{j+1}}>0,
$$

for $s \in(k / 2, k-2]$. (The monotonicity at $k / 2$ follows by continuity).

For the monotonity of $Z_{j}$, we note that (16) implies

$$
Z_{j+1}(s)=\sum_{r \geq 1} \Lambda(r) \log ^{j}(r) \cdot\left(\frac{(-1)^{j+1}}{r^{s}}+\frac{1}{r^{k-s}}\right)>0
$$

for all $j$. The positivity of each term follows trivially for $j$ odd and from the inequality $r^{s}>r^{k-s}$ when $j$ is even 
Returning to the proof of the theorem, we see that

$$
\begin{aligned}
& q_{f}^{m}(z)= \\
& \sum_{n=k / 4-1}^{k / 2-2}\left(\begin{array}{c}
k-2 \\
2 n+1
\end{array}\right) \delta_{n} \widetilde{\Lambda}_{f}(2 n+2)\left(\sum^{*} c_{j_{1}, \ldots, k_{1}, \ldots, n_{j_{1}}, \ldots, n_{k_{1}} \ldots} \Psi_{j_{1}}^{n_{j_{1}}}(2 n+2) \ldots Z_{k_{1}}^{n_{k_{1}}}(2 n+2) \ldots\right) z^{n} \\
& \frac{2(k-2) !}{(2 \pi)^{k}} \sum_{n=k / 4-1}^{k / 2-2} \delta_{n} a_{n}\left(\sum^{*} c_{j_{1}, \ldots, k_{1}, \ldots, n_{j_{1}}, \ldots, n_{k_{1}} \ldots} \Psi_{j_{1}}^{n_{j_{1}}}(2 n+2) \ldots Z_{k_{1}}^{n_{k_{1}}}(2 n+2) \ldots\right) z^{n}, \quad(19)
\end{aligned}
$$

where $a_{n}=\zeta(2 n+2) \zeta(k-2 n-2)$. As shown by (17), $a_{n}$ and thus $\delta_{n} a_{n}$ is increasing as $n$ ranges from $k / 4-1$ to $k / 2-2$. Also, the term inside the brackets is a linear combination, with positive coefficients, of products of $Z_{j}$ and $\Psi_{j}$ which, by Lemma 4.4 , are increasing in the range of interest. Therefore, the coefficients of $z^{n}$ form an increasing and non-negative sequence and thus, by the Eneström-Kakeya Theorem we deduce the result.

\section{Proof of Proposition 1.2 and discussion}

We begin with the proof of the initial cases of part i). of Conjecture 1.1.

\subsection{Proof of Proposition 1.2}

This numerical verification relies on an old theorem of Cohn [5] (see also the proof and formulation in [4]).

Theorem 5.1 (Cohn). Suppose that $P(z)$ is a self-inversive polynomial; that is, $P(z)=$ $\varepsilon z^{d} \bar{P}(1 / z)=: P^{*}(z)$ for $|\varepsilon|=1$ where - denotes complex conjugation of the coefficients. Then $P(z)$ and $\left(P^{\prime}(z)\right)^{*}$ have the same number of zeros inside the open unit disk $|z|<1$. The polynomial $P(z)$ has all its roots on the unit circle if and only if the zeros of the derivative $P^{\prime}(z)$ lie in the closed unit disk $|z| \leq 1$.

Although this theorem can be difficult to use for infinite families, it is well-suited to check a finite number of cases. In this way, the conjecture for the full period polynomials was checked for derivatives $m \leq 3$ and all newforms of level 1 and weight $k \leq 50$. We note that care must be taken when performing this calculation, as when the weight grows the expressions involved quickly involve very large numbers multiplied by very small numbers, and so high precision must be used in order to obtain numerical stability. The necessary commands for $L$-functions, their completions, their derivatives, and of course for root finding, are all built directly into Magma.

For the odd parts of period polynomials, there is by definition a root at $z=0$. The same numerical verifications confirm for $m \leq 3$ and $k \leq 50$ that the number of zeros of the odd part of the period polynomial divided by $z$ (which eliminates the known zero at $z=0$ ) inside the open unit disk is the same as the number of zeros of that polynomial differentiated 
and then with reciprocated coefficients. Computations have verified in this range that there are precisely two such zeros. As the odd part of the period polynomial is self-reciprocal and odd the zeros come in pairs under negation, complex conjugation, and reciprocating absolute values. Thus, the only possibility is that there is the trivial zero at $z=0$ and a pair of roots $\pm b$ which are real and have $|b|<1$. Then there are also roots at $\pm 1 / b$ where $1 / b>1$, and the remainder of roots must lie on the unit circle.

\subsection{Discussion}

As commented above, this result seems somewhat surprising, as usually the "interesting" information of modular $L$-functions is in their first non-vanishing central derivative, and no such restriction is made here in the study of these higher $L$-derivative values. Significant theoretical bounds seem to be required to prove this conjecture, even in the first unproven case of $m=1$, for example. In particular, proofs like those in [20] require non-negativity results for central $L$-values, which seem to be harder for higher derivatives. However, inspired by the theory of super-positivity in Yun and Zhang's breakthrough work on higher derivative Gross-Zagier formulas in the function field setting [31], Goldfeld and Huang [16] proved that infinitely many modular forms do satisfy this property, which would be required for a proof in the style of [20] to work in our case.

Further, it would also be interesting to present, even in the known case of $m=0$, a uniform proof which covers all cases simultaneously without breaking into finitely many cases and checking the remaining ones numerically. This would be nice theoretically, in order to understand the "reason" why the previous results on period polynomials are true, and would be important in order to prove a general conjecture as stated here, where one would like to study infinitely many iterated derivatives (and so cannot numerically verify finitely many cases on each).

Recall also that, as stated above, it also appears that the odd part

$$
\sum_{\substack{n=1 \\
n \text { odd }}}^{k-3}\left(\begin{array}{c}
k-2 \\
n
\end{array}\right) i^{1-n} \Lambda_{f}^{(m)}(n+1) z^{k-2-n}
$$

has all of its zeros on the unit circle, except for trivial zeros at the points $0, \pm a, \pm 1 / a$ for some real number $a$. In [6], this conjectured behavior for the odd part is shown to be true when $m=0$ with $a=2$. There, it is shown that these "trivial zeros" arise in a natural way from the Eichler-Shimura relations. In addition to our proof of the "odd polynomial" version of the conjecture in the special case of Eisenstein series and $m=0$ and $m=1$, there is numerical evidence for the truth of the full conjecture.

As mentioned in the introduction, results on the zeros of (classical) period polynomials for cusp forms in [6, 14, 20] etc. extend analogous results about Eisenstein series in [24, 27]. Furthermore, these results, taken together, cover both the "odd" part and the full period polynomial, just as our conjecture does. 


\section{$5.3 \quad$ Examples}

We now describe several numerical examples of our conjecture for context. As shown in [20], the zeros of period polynomials equidistribute on the unit circle for large weights $k$. However, in small weights, this behavior is not yet exhibited. For instance, the period polynomial for $\Delta \in S_{12}$ has the following roots:

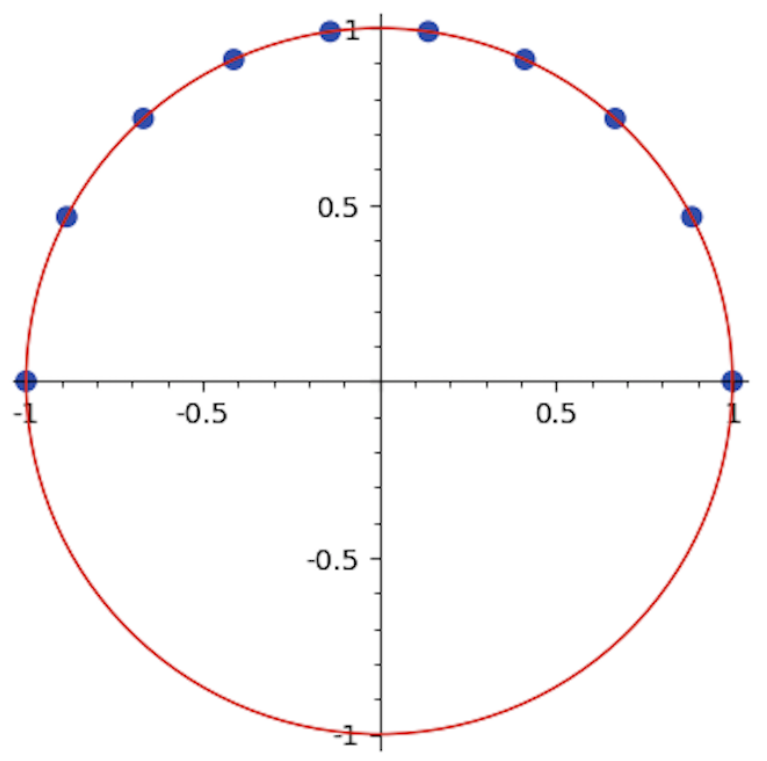

Remarkably, they all lie on the upper half of the unit circle. For $\Delta$ again, the first derivative period polynomial has similar-looking roots, but where the top two roots seem to migrate to the top of the circle and split up into $\pm i$ :

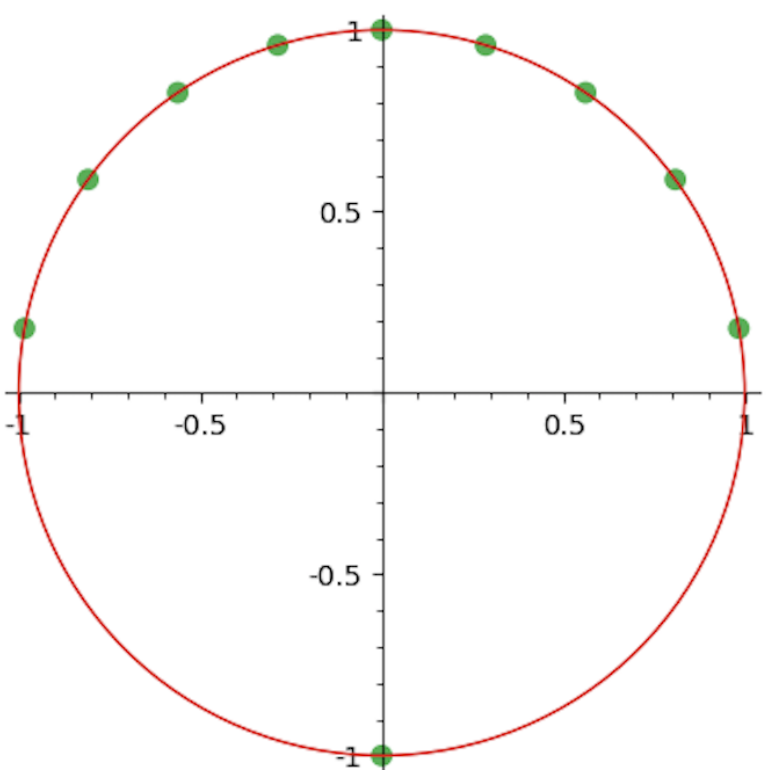


For the next two derivative period functions ( $m=2,3$ for $\Delta$ ), similar locations of the roots are observed, but there seems to be a behavior depending on the parity of the number of derivatives taken, presumably resulting from the sign of the functional equations for $\Lambda_{\Delta}^{(m)}$ :
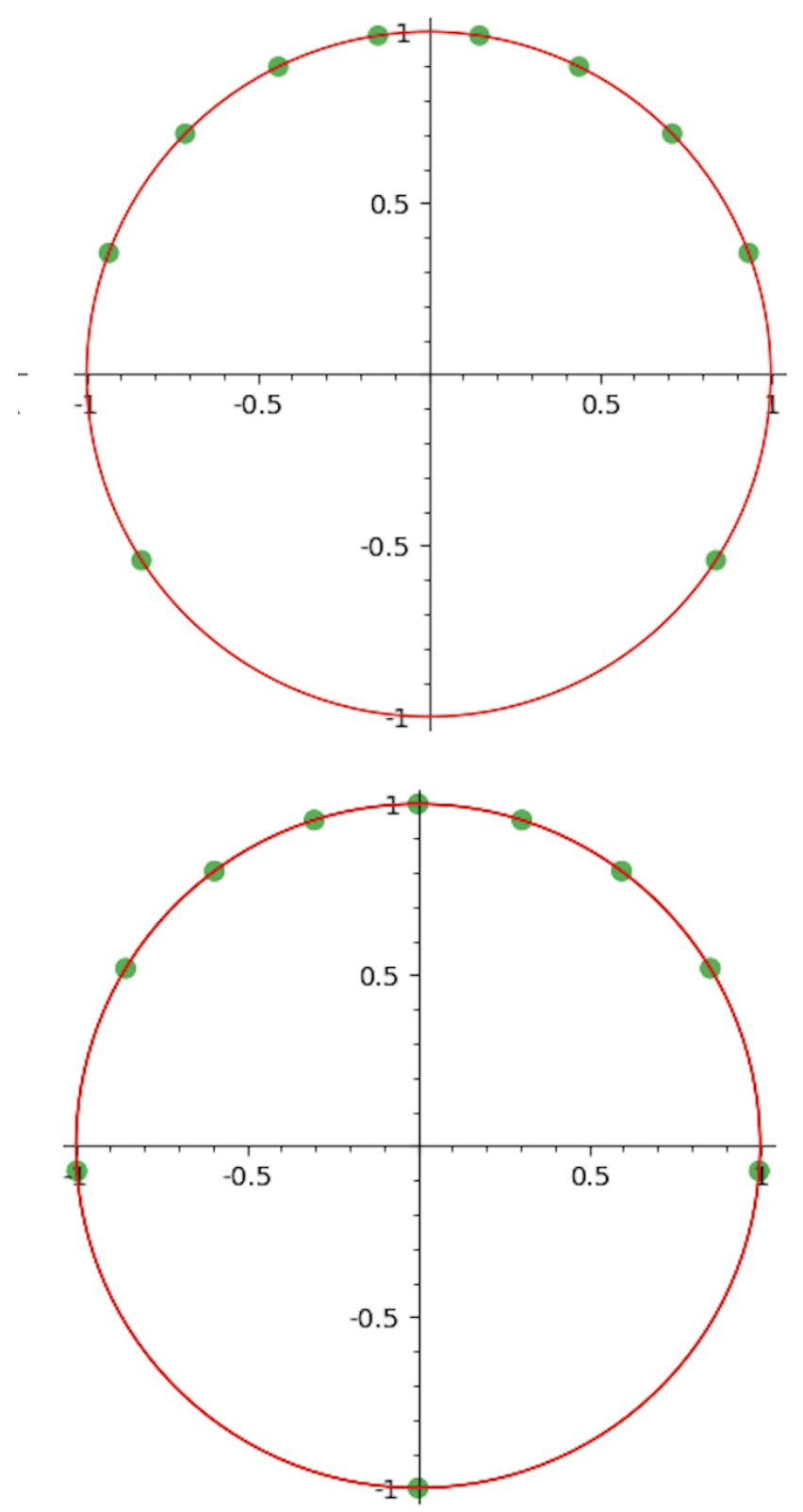

As an example of the behavior of the number $a$ in part ii). of Conjecture 1.1, we recall the example of the normalized weight 20, level 1 cuspidal eigenform $f$. A rescaling and change of variables in the second polynomial in Conjecture 1.1 yields a polynomial approximately given by

$$
z^{16}-5.805 z^{14}+9.685 z^{12}-6.720 z^{10}+6.720 z^{6}-9.685 z^{4}+5.805 z^{2}-1 .
$$


The zeros predicted by Conjecture 1.1 in this case are on the unit circle with arguments approximately $0,13.5$ and 43 degrees, together with the real and complex conjugates of these points, and seems to have zeros at $\pm a^{ \pm 1}$ for $a$ approximately 1.9.

Finally, we describe, following a suggestion of Don Zagier, numerical experiments on perturbations of Conjecture 1.1. In an email to Ken Ono which has been shared with the authors [33], Zagier sought out to uncover how "special" the Riemann Hypothesis for Period Polynomials of [20] is, in the following sense. To what extent do generic polynomials which "look like" period polynomials also have all their roots on the unit circle? The proof of [20] shows that the roots are more and more "likely" to lie on the unit circle as the weight grows, based on their analytic proof that it holds for all but finitely many weights, but a number of small cases have to be checked by hand. Zagier's question is whether these small cases very "easily" hold or not. Of course, it is important that one considers self-inversive polynomials, but beyond this restriction, how close are period polynomials to the edge of the domain of polynomials with roots on the unit circle? To make this precise, Zagier considers splitting the period polynomials into even and odd parts, and inserting a generic real multiple in front of the odd part of the period polynomial. This is natural both due to Manin's Period Theorem which shows that these parts are both algebraic up to a fixed transcendental multiple, and due to the root behavior of the odd part of the period polynomial discussed again.

Here, we repeat Zagier's experiment for the function $\Delta \in S_{12}$ (which was also the case he considered). We will use a different normalization than his, since we don't have the same rational structure of the period polynomials to work with in higher derivatives which he utilized in his formulas, but overall this only changes things by multiplying the intervals in question by a constant. We split $Q(m ; z):=Q_{f}(m ; z)$ into its even and odd parts $Q(m ; z)=$ : $Q^{+}(m ; z)+Q^{-}(m ; z)$. Then consider the perturbed family of polynomials

$$
Q_{t}(m ; z):=Q^{+}(m ; z)+t \cdot Q^{-}(m ; z)
$$

for real values of $t$. Of course, by choice, $Q_{t}(m ; z)$ is still self-inversive for every choice of $t$. The Riemann Hypothesis for Period Polynomials states that $Q_{1}(m ; z)$ has roots on the unit circle. For which other values of $t$ is this true? It turns out it is only true in a very narrow interval very nearly centered around $t=1$. Computationally, this interval is approximately $[0.999963,1.000024]$. This means that the period polynomial for $\Delta$ in some sense "barely" has all its roots on the unit circle.

In the following table, we record the results of numerical experiments illustrating this behavior for the first three derivative period polynomials of $\Delta$ as well, and the ranges $\left[t^{-}, t^{+}\right]$ where they satisfy the Riemann Hypothesis.

\subsection{Future work}

We conclude with a list of questions and problems which may be interesting for future work.

1. As shown in Table 1, it seems that the numbers $a$ in part ii). of Conjecture 1.1 almost only depend on $k$ and $m$. It would be interesting to see if similar Eichler-Shimura-type 
Table 2: Ranges where perturbed "derivative period poly's" of $\Delta$ have roots on the unit circle

\begin{tabular}{|c|c|c|}
\hline Number of derivatives taken & Approximate value of $t^{-}$ & Approximate value of $t^{+}$ \\
\hline 0 & 0.999963 & 1.000024 \\
\hline 1 & 0.9996284 & 1.000734 \\
\hline 2 & 0.9997141 & 1.000168 \\
\hline 3 & 0.9989016 & 1.001929 \\
\hline
\end{tabular}

relations which give rise to the value $a=2$ in the classical period polynomial case also explain this numerical observation.

2. It would also be interesting to describe the consequences for Eichler-Shimura cohomology. In particular, what are the applications of our theorems above and of the conjecture in the case of cusp forms?

3. Is there a uniform proof of the Riemann Hypothesis for Period Polynomials of [20] which "explains" the small weight examples like $\Delta$ and why they satisfy the strong conditions required to have roots on the unit circle as indicated by Zagier's experiment?

4. Is there a suitable theory of Manin "zeta-polynomials" as discussed in the ordinary period polynomial case in [28] following the proof of the Riemann Hypothesis for Period Polynomials?

5. There seem to be several phenomena related to the parity of the number of derivatives taken. For instance, the widths of the intervals in Table 2, and the pictures of the root locations in the examples for $\Delta$ above. Do these have explanations arising directly from the sign of the functional equation of $\Lambda_{f}^{(m)}$ ?

\section{References}

[1] B. Berndt, A. Straub Ramanujan's Formula for $\zeta(2 n+1)$, Exploring the Riemann Zeta Function, 190 years from Riemann's Birth, Springer, editors: H. Montgomery, A. Nikeghbali, and M. Rassias, 2017, pages 13-34.

[2] R. Bruggeman, Y. Choie, and N. Diamantis, Holomorphic automorphic forms and cohomology, Memoirs AMS 253, 1212, 2018.

[3] F. Brown, Multiple Modular Values and the relative completion of the fundamental group of $M_{1,1}$, preprint, arXiv:1407.5167.

[4] F. F. Bonsall and M. Marden, Zeros of Self-inversive Polynomials, Proc. Amer. Math. Soc. 3 (1952), 471-475. 
[5] A. Cohn, Über die Anzahl der Wurzeln diner algebraischen Gleichung in einem Kreise, Math. Z. 14 (1922), 110-148.

[6] J.B. Conrey, D.W. Farmer, and Ö. Imamoğlu, The nontrivial zeros of period polynomials of modular forms lie on the unit circle, Int. Math. Res. Not. no. 20, 4758-4771 (2013).

[7] P. Deligne Valeurs de Fonctions L et périodes dintégrales, Proceedings of Symposia in Pure Mathematics 33, 313-346 (1979).

[8] N. Diamantis, Special values of higher derivatives of L-functions, Forum Math. 11 no. 1, 229-252 (1999).

[9] N. Diamantis, Hecke Operators and Derivatives of L-Functions, Compositio Math. 125 no. 1, 39-54 (2001).

[10] N. Diamantis, The geometry of certain cocycles associated to derivatives of L-functions, Forum Math. 17 no. 5, 739-752 (2005).

[11] N. Diamantis, M. Neururer, F. Strömberg, A correspondence of modular forms and applications to values of L-series, Res. number theory (2015) 1: 27

[12] N. Diamantis, C. O'Sullivan, The dimensions of spaces of holomorphic second-order automorphic forms and their cohomology, Trans. of the AMS, Vol. 360, Number 11, 5629-5666.

[13] G. Eneström, Ramarque sur un théorème relatif aux recines de l'equation $a_{n} x_{n}+\cdots+a_{0}=$ 0 où tous les coefficients sont réels et positifs, Tôhoku Math. J. 18, 34-36 (1920), translation of a Swedish article in Ofversigt of Konogl. Vertenskaps Akademiens Förhandlingar 50, 405-415 (1893).

[14] A. El-Guindy, W. Raji, Unimodularity of zeros of period polynomials of Hecke eigenforms, Bull. Lond. Math. Soc. 46 no. 3, 528-536 (2014).

[15] D. Goldfeld, Special values of derivatives of L-functions, Number theory (Halifax, NS, 1994), 159-173, CMS Conf. Proc., 15, Amer. Math. Soc., Providence, RI (1995).

[16] D. Goldfeld and B. Huang, Super-positivity of a family of L-functions, arXiv:1612.09359 (2016).

[17] B. Gross and D. Zagier Heegner points and derivative of L-series Invent. Math. 85, 225-320 (1986).

[18] E. Grosswald, Die Werte der Riemannschen Zeta-funktion an ungeraden Argumentstellen Nachr. Akad. Wiss. Göttingen (1970), 9-13.

[19] H. Iwaniec, Topics in classical automorphic forms Graduate Studies in Mathematics, Vol. 17, AMS, 1991. 
[20] S. Jin, W. Ma, K. Ono, and K. Soundararajan, The Riemann Hypothesis for period polynomials of modular forms, Proc. Natl. Acad. of Sci. U.S.A. 113 no. 10, 2603-2608 (2016).

[21] S. Kakeya, On the limits of the roots of an algebraic equation with positive coefficients, Tohoku Math. J. 2, 140-142 (1912-1913).

[22] W. Kohnen and D. Zagier, Modular forms with rational periods in Modular Forms, R.A. Rankin (ed.), Ellis Horwood, Chichester 197-249 (1984)

[23] M. Kontsevich and D. Zagier, Periods, Mathematics unlimited -2001 and beyond, 771808, Springer, Berlin, 2001.

[24] M. Lalín and C. Smyth, Unimodularity of zeros of self-inversive polynomials Acta Math. Hungar. 138 (2013), no. 1-2, 85-101. Addendum, Acta Math. Hungar. 147 (2015), no. 1, $255-257$.

[25] Y. T. Manin, Periods of parabolic points and p-adic Hecke series, Math. Sb., 371-393 (1973).

[26] Y. I. Manin, Local zeta factors and geometries under Spec Z, Izv. Russian Acad. Sci. (Volume dedicated to J.-P. Serre) 80 no. 4, 123-130 (2016)

[27] M. Murty, C. Smyth, and R. Wang, Zeros of Ramanujan polynomials J. of the Ramanujan Math. Soc. 26, 107-125 (2011).

[28] K. Ono, L. Rolen, and F. Sprung, Zeta-polynomials for modular form periods, Adv. Math. 306, 328-343 (2017).

[29] F. Olver, D. Lozier, R. Boisvert, and C. Clark, NIST handbook of mathematical functions U.S. Department of Commerce, National Institute of Standards and Technology, Washington, DC; Cambridge University Press, Cambridge, 2010.

[30] H. Rademacher, Zur Theorie der Modulfunktionen J. Reine Angew. Math. 167, 312 - 336 (1932).

[31] Z. Yun and W. Zhang, Shtukas and the Taylor expansion of L-functions, Ann. Math. 186 (2017), no. 3, 767-911.

[32] D. Zagier, Periods of modular forms and Jacobi theta functions, Invent. Math. 104 no. 3 449-465 (1991).

[33] D. Zagier, Personal communication to Ken Ono. 